\title{
Nível de infestação de Diaphorina citri no Município de Inconfidentes, Minas Gerais
}

\author{
Bruna Melo da Silva ${ }^{1}$, Jamil de Morais Pereira ${ }^{1} \&$ Luis Carlos Dias da Rocha ${ }^{1}$
}

\section{RESUMO}

Os citros são plantas de origem asiática, que se adaptaram muito bem as condições climáticas e ambientais brasileiras. Possuem grande importância econômica e social, movimentando empregos direto e indiretamente. Entretanto, por ser uma planta introduzida, vieram com ela, alguns insetos e micro-organismos que frequentemente atingem o status de pragas/doenças, e que se não controladas adequadamente podem ocasionar prejuízos aos agricultores. Dentre as doenças destaca-se a Huanglongbing (HLB), conhecida popularmente como greening que causa sérios danos à produção. Objetivou-se com este trabalho, realizar um levantamento do vetor Diaphorina citri nos pomares citrícolas comerciais e em plantas de murta das vias públicas do Município de Inconfidentes - MG e verificar em quais áreas o inseto se encontrava mais concentrado, a fim de lançar estratégias de manejo, definindo áreas prioritárias para o controle. A metodologia foi dividida em três etapas: Na primeira etapa foi feita a análise dos pomares citrícolas, em que foram selecionadas quatro propriedades produtoras da variedade Ponkane Valência, formadas por sete pomares. Realizou-se o levantamento do vetor: Diaphorina citri nos pomares do município, em que o estudo abrangeu uma área de $1250 \mathrm{~m}^{2}$, para cada pomar, distribuída em 3 pontos espaçados a cada $50 \mathrm{~m}$. Em cada ponto foi colocado uma armadilha adesiva de cor amarela a uma altura de 1,6m. Cada armadilha foi trocada a cada 15 dias, sendo coletado 25 brotações em cada propriedade, num total de 100 brotações por amostra. Na segunda etapa foi realizado o levantamento do inseto nas plantas de murta constituintes da urbanização da cidade. Já a terceira etapa consistiu na manipulação dos dados no software Arc gis Pro. Ao todo foram quantificados 27 adultos, 221 ovos e 248 ninfas. As plantas de murta $4 ; 5 ; 6 ; 10 ; 21$ e 28 foram as que obtiveram maiores médias de ninfas, ovos e adultos, se identificando como áreas prioritárias ao controle. Embora o inseto esteja presente nas plantas de murta do paisagismo da cidade, este não foi identificado nos pomares, mesmo em diagnósticos positivos a doença. Conclui-se que os resultados obtidos com o trabalho é um alerta para uma possível infestação do Diaphorina citri em pomares citrícolas da região, ao qual, deve-se iniciar um monitoramento periódico destes pomares, e mesmo que não se encontre o inseto nos pomares indica-se o controle, visto que se trata de um vetor e que o monitoramento com armadilha adesiva amarela, mesmo sendo o mais eficiente dos métodos de monitoramento, ainda é falho.

Termos de indexação: citricultura, huanglongbing, HLB, greening.

\footnotetext{
${ }^{1}$ Instituto Federal de Educação, Ciências e Tecnologia do Sul de Minas Gerais (IFSULDEMINAS), Campus Inconfidentes, Ouro Fino, MG, Brasil

Autor correspondente: Bruna Melo da Silva, Instituto Federal de Educação, Ciência e Tecnologia do Sul de Minas Gerais (IFSULDEMINAS), Campus Inconfidentes, Rua Tocantins, 77, CEP 37570-000, Ouro Fino, MG, Brasil. E-mail: bruh29cats@gmail.com
} 


\section{Infestation level of Diaphorina citri in the Municipality of Inconfidentes, Minas Gerais State, Brazil}

\section{SUMMARY}

Citrus are asian plants that have adapted very well to brazilian climatic and environmental conditions. They have great economic and social importance, moving jobs directly and indirectly. However, because it is an introduced plant, some insects and microorganisms that often reach the status of pests/diseases have come with it, and if not properly controlled they can cause harm to farmers. Among the diseases stands out huanglongbing (HLB), popularly known as greening that causes serious damage to production. The objective of this work was to survey the Diaphorina citri vector in the commercial citrus orchards and myrtle plants of the Inconfidentes Municipality, Minas Gerais State, Brazil, and to verify in which areas the insect was most concentrated, in order to launch strategies of management, defining priority areas for control. The methodology was divided in three stages: in the first stage the citrus orchards were analyzed, in which four producing properties of the Ponkan tangerine and Valencia sweet orange varieties were selected, formed by seven orchards. A survey of the vector: Diaphorina citri was carried out in the orchards of the municipality, in which the study covered an area of $1250 \mathrm{~m}^{2}$ for each orchard, distributed in 3 spaced spots every $50 \mathrm{~m}$. At each point a yellow colored adhesive trap was placed at a height of $1.6 \mathrm{~m}$. Each trap was changed every 15 days, and 25 shoots were collected in each plot, for a total of 100 shoots per sample. In the second stage the insect survey was carried out on the myrtle plants that constituted the urbanization of the city. The third step consisted in manipulating the data in the software Arc gis Pro. In all, 27 adults, 221 eggs and 248 nymphs were quantified. The myrtle plants $4 ; 5 ; 6 ; 10 ; 21$ and 28 were the ones that obtained higher averages of nymphs, eggs and adults, identifying themselves as priority areas for control. Although the insect is present in the myrtle plants of the city's landscaping, it was not identified in the orchards, even in positive diagnoses of the disease. It is concluded that the results obtained with the work is an alert for a possible infestation of Diaphorina citri in citrus orchards of the region, to which a periodic monitoring of these orchards should be initiated, and even if the insect is not found in the orchards indicates the control, since it is a vector and that monitoring with a yellow adhesive trap, even being the most efficient of the monitoring methods, is still flawed.

Index terms: citriculture, huanglongbing, HLB, greening.

\section{INTRODUÇÃO}

O aparecimento de pragas e doenças na citricultura não é uma novidade, mas atualmente a produção dos citros vem sendo fortemente ameaçada pelo Huanglongbing, (HLB), uma doença muito agressiva e destrutiva, causada por bactérias do gênero Liberibacter spp (Paiva, 2009), cujo o vetor é a Diaphorina citri, Hemiptera: Liviidae (Burckhardt \& Ouvrard, 2012).

Considerada a principal doença da citricultura mundial (Tomaseto, 2016) o HLB, causa a queda prematura dos frutos, diminuindo a produtividade e o suco perde o seu valor comercial. Tal patologia pode ser identificada pela alteração da aparência das plantas, que quando infectadas, ficam com ramos amarelados, em comparação com os ramos sadios de coloração verde (Atihe Junior et al., 2006).

Assim as folhas apresentam mosqueado que contrasta amarelo e verde e, em alguns casos, engrossamento e clareamento das nervuras (Atihe Junior et al., 2006). Os frutos se apresentam assimétricos e deformados, podendo surgir manchas arredondadas e amareladas na casca, com abortamento de sementes (Fernandes, 2004; Atihe Junior et al., 2006). Embora o vetor D. citri seja o principal responsável pela transmissão da doença no Brasil, esta pode ocorrer por contato da planta sadia com a planta doente através da enxertia (Atihe Junior et al., 2006).

O Brasil é responsável por cerca de $50 \%$ da produção mundial de suco de laranja e exporta $98 \%$ do que produz, alcançando $85 \%$ de participação no mercado mundial (Erpen et al., 2018); Todavia, desde o primeiro relato da presença da doença no Brasil em 2004, na região de Araraquara, no Estado de São Paulo (Coletta et al., 2004) a patologia vem preocupando produtores de todo o país, inclusive os residentes no município de Inconfidentes, interior do Estado de Minas Gerais, tendo a patologia sido registrada em Agosto de 2017 (Instituto Mineiro de Agropecuária, (IMA) dados não publicados).

No Município de Inconfidentes, foram identificadas quatro propriedades comerciais de citros segundo os registros fornecidos pelo IMA. O Município, detém uma 
área colhida de 14 hectares de Tangerina Ponkan, com uma produção de 280 toneladas em 2016, aproximadamente 20 toneladas produzidas por hectare (Empresa Brasileira de Pesquisa Agropecuária, 2016). Porém o crescimento econômico na área cítrica da região pode estar ameaçado, dado o registro do greening e seu possível agravamento caso seja constatada a presença do vetor $D$. citri no município. Tal hipótese não está descartada, uma vez que as vias públicas do município apresentam exemplares de Murraya paniculata L.Jack, considerada a planta hospedeira preferida pelo inseto (Fernandes, 2004; Parra et al., 2010).

Nesse sentido, este trabalho tem como objetivo: realizar um levantamento do vetor $D$. citri nos pomares citrícolas comerciais e em plantas de murta das vias públicas do Município de Inconfidentes e verificar em quais áreas o inseto se encontra mais concentrado.

\section{MATERIAL E MÉTODOS}

O estudo foi desenvolvido no período de maio à setembro de 2018, nos pomares e em plantas de 'murtas' presentes na arborização das vias públicas, do município de Inconfidentes, no Sul do Estado de Minas Gerais. O município, está localizado sob a latitude $22^{\circ} 19^{\prime} 01$ ' S e altitude $46^{\circ} 19^{\prime} 40^{\prime \prime} \mathrm{W}$ se identificando como área apta para o cultivo de citros, devido a sua precipitação anual estar entre 600 e $1300 \mathrm{~mm}$ e temperaturas maiores que $18^{\circ} \mathrm{C}$ e menores que $31^{\circ} \mathrm{C}$ (Minas Gerais, 2011).

A metodologia consistiu em três etapas: monitoramento de D. citri nos pomares citrícolas do município, monitoramento de $D$. citri nas plantas de 'murta' presentes nas vias públicas do município e manipulação dos dados estatísticos.

\section{Monitoramento de $D$. Citri nos pomares citricolas do município}

Foram selecionadas 4 propriedades, com base nos documentos internos fornecidos pelo IMA. Aqui denominadas de propriedade A, B, C e D. Nestas foram identificados 7 pomares, com idades variando de 1 a 25 anos, sendo 6 em produção (Figura 1).

Os espaçamentos de plantio variam de 3,0 metros na entrelinha $x 3,0$ metros entre plantas a 3,5 metros na entrelinha x 3,5 metros entre plantas. As copas utilizadas foram de Laranja Valência ou Ponkan e os porta- enxertos foram limão Cravo ou Tangerina Cleópatra.

Delimitou-se uma área de $1250 \mathrm{~m}^{2}$ para cada pomar, calculada pelo programa do Google Eath Pro. A área

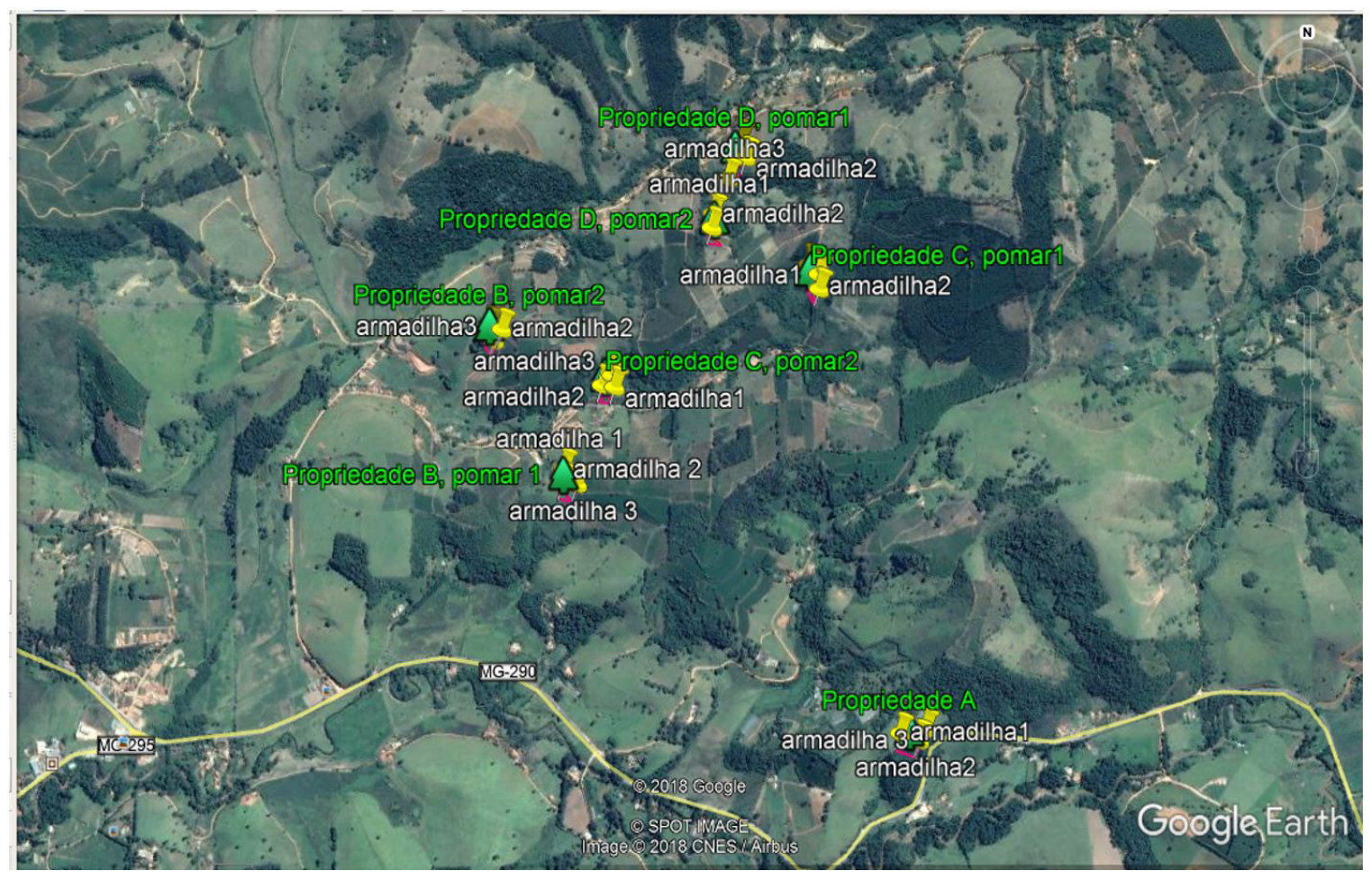

Figura 1. Localização dos pomares citrícolas no município de Inconfidentes, estado de Minas Gerais (MG). 
foi distribuída em 3 pontos, priorizando pontos de marcação, próximos a matas, ou a lagos, represas e açudes, que favorecem a captura do inseto, segundo Yamamoto et al. (2015), ou ainda próximos a plantações. Em cada ponto de coleta foi instalada uma armadilha adesiva amarela de dimensões: $23 \mathrm{~cm}$ de altura e 11 $\mathrm{cm}$ de largura, posicionada em uma árvore a 1,6 $\mathrm{m}$ de altura. Estas armadilhas ficaram distanciadas de $50 \mathrm{~m}$.

Todas as armadilhas foram georeferenciadas e identificadas por uma série de algarismos. 1.1.1.1 a 1.n.n.n, onde o $1^{\circ}$ algarismo representa o município de Inconfidentes, o $2^{\circ}$ algarismo a região pesquisada
(Propriedade), o $3^{\circ}$ algarismo o pomar e o $4^{\circ}$, a localização da armadilha em uma determinada planta (Tabela 1).

As armadilhas foram trocadas a cada 15 dias e levadas ao laboratório de Agroecologia do IFSULDEMINAS- Campus Inconfidentes para identificação e quantificação dos insetos adultos coletados. Tal observação foi realizada, utilizando uma lupa de aumento de 20 vezes.

A cada troca de armadilha foram coletadas 25 brotações aleatórias, em cada propriedade, totalizando 100 brotações. As brotações foram colocadas em recipientes de plástico com $13 \mathrm{~cm}$ de comprimento e levadas ao laboratório para a identificação e a quantificação de ovos e ninfas.

Tabela 1. Localização e caracterização das áreas localizadas nas quatro regiões pesquisadas

\begin{tabular}{|c|c|c|c|c|c|c|c|}
\hline \multirow{2}{*}{ Código } & \multirow{2}{*}{ Propriedade } & \multirow{2}{*}{ Pomar } & \multirow{2}{*}{ Município } & \multicolumn{2}{|c|}{ Coordenadas Geográficas } & \multirow{2}{*}{ Idade } & \multirow{2}{*}{ Variedades } \\
\hline & & & & W & $\mathrm{S}$ & & \\
\hline 1.1 .1 .1 & A & Pomar1 & Inconfidentes & $046^{\circ} 17^{\prime} 09.8^{\prime \prime}$ & $22^{\circ} 18^{\prime} 38.3^{\prime \prime}$ & 13 & $\begin{array}{l}\text { Ponkan e } \\
\text { Valência }\end{array}$ \\
\hline 1.1.1.2 & A & Pomar1 & Inconfidentes & $046^{\circ} 17^{\prime} 07.3^{\prime \prime}$ & $22^{\circ} 18^{\prime} 39.2^{\prime \prime}$ & 13 & $\begin{array}{l}\text { Ponkan e } \\
\text { Valência }\end{array}$ \\
\hline 1.1.1.3 & A & Pomar1 & Inconfidentes & $046^{\circ} 17^{\prime} 06.5^{\prime \prime}$ & $22^{\circ} 18^{\prime} 37.9^{\prime \prime}$ & 13 & $\begin{array}{l}\text { Ponkan e } \\
\text { Valência }\end{array}$ \\
\hline 1.2.1.1 & B & Pomar2 & Inconfidentes & $046^{\circ} 17^{\prime} 52.5^{\prime \prime}$ & $22^{\circ} 18^{\prime} 08.9^{\prime \prime}$ & 1 & Ponkan \\
\hline 1.2.1.2 & B & Pomar2 & Inconfidentes & $046^{\circ} 17^{\prime} 52.6^{\prime \prime}$ & $22^{\circ} 18^{\prime} 10.5^{\prime \prime}$ & 1 & Ponkan \\
\hline 1.2.1.3 & B & Pomar2 & Inconfidentes & $046^{\circ} 17^{\prime} 50.8^{\prime \prime}$ & $22^{\circ} 18^{\prime} 10.6^{\prime \prime}$ & 1 & Ponkan \\
\hline 1.2.2.1 & B & Pomar3 & Inconfidentes & $046^{\circ} 18^{\prime} 02.1^{\prime \prime}$ & $22^{\circ} 17^{\prime} 53.3^{\prime \prime}$ & 13 & $\begin{array}{l}\text { Ponkan e } \\
\text { Valência }\end{array}$ \\
\hline 1.2.2.2 & B & Pomar3 & Inconfidentes & $046^{\circ} 18^{\prime} 00.4^{\prime \prime}$ & $22^{\circ} 17^{\prime} 53.5^{\prime \prime}$ & 13 & $\begin{array}{l}\text { Ponkan e } \\
\text { Valência }\end{array}$ \\
\hline 1.2.2.3 & $\mathrm{B}$ & Pomar3 & Inconfidentes & $046^{\circ} 18^{\prime} 01.4^{\prime \prime}$ & $22^{\circ} 17^{\prime} 54.7^{\prime \prime}$ & 13 & $\begin{array}{l}\text { Ponkan e } \\
\text { Valência }\end{array}$ \\
\hline 1.3.1.1 & $\mathrm{C}$ & Pomar4 & Inconfidentes & $046^{\circ} 17^{\prime} 20.3^{\prime \prime}$ & $22^{\circ} 17^{\prime} 49.2^{\prime \prime}$ & 23 & $\begin{array}{l}\text { Ponkan e } \\
\text { Valência }\end{array}$ \\
\hline 1.3.1.2 & $\mathrm{C}$ & Pomar4 & Inconfidentes & $046^{\circ} 17^{\prime} 20.4^{\prime \prime}$ & $22^{\circ} 17^{\prime} 47.6^{\prime \prime}$ & 23 & $\begin{array}{l}\text { Ponkan e } \\
\text { Valência }\end{array}$ \\
\hline 1.3.1.3 & $\mathrm{C}$ & Pomar4 & Inconfidentes & $046^{\circ} 17^{\prime} 21.7^{\prime \prime}$ & $22^{\circ} 17^{\prime} 46.6^{\prime \prime}$ & 23 & $\begin{array}{l}\text { Ponkan e } \\
\text { Valência }\end{array}$ \\
\hline 1.3.2.1 & $\mathrm{C}$ & Pomar5 & Inconfidentes & $046^{\circ} 17^{\prime} 46.1^{\prime \prime}$ & $22^{\circ} 17^{\prime} 59.9^{\prime \prime}$ & 25 & $\begin{array}{l}\text { Ponkan e } \\
\text { Valência }\end{array}$ \\
\hline 1.3.2.2 & $\mathrm{C}$ & Pomar5 & Inconfidentes & $046^{\circ} 17^{\prime} 47.7^{\prime \prime}$ & $22^{\circ} 17^{\prime} 59.7^{\prime \prime}$ & 25 & $\begin{array}{l}\text { Ponkan e } \\
\text { Valência }\end{array}$ \\
\hline 1.3.2.3 & $\mathrm{C}$ & Pomar5 & Inconfidentes & $046^{\circ} 17^{\prime} 46.4^{\prime \prime}$ & $22^{\circ} 17^{\prime} 58.6^{\prime \prime}$ & 25 & $\begin{array}{l}\text { Ponkan e } \\
\text { Valência }\end{array}$ \\
\hline 1.3.3.1 & $\mathrm{D}$ & Pomar6 & Inconfidentes & $046^{\circ} 17^{\prime} 30.0^{\prime \prime}$ & $22^{\circ} 17^{\prime} 32.6^{\prime \prime}$ & 3 & Ponkan \\
\hline 1.3.3.2 & $\mathrm{D}$ & Pomar6 & Inconfidentes & $046^{\circ} 17^{\prime} 29.5^{\prime \prime}$ & $22^{\circ} 17^{\prime} 34.5^{\prime \prime}$ & 3 & Ponkan \\
\hline 1.3.3.3 & $\mathrm{D}$ & Pomar6 & Inconfidentes & $046^{\circ} 17^{\prime} 31.7^{\prime \prime}$ & $22^{\circ} 17^{\prime} 36.8^{\prime \prime}$ & 3 & Ponkan \\
\hline 1.3.4.1 & $\mathrm{D}$ & Pomar7 & Inconfidentes & $046^{\circ} 17^{\prime} 33.6^{\prime \prime}$ & $22^{\circ} 17^{\prime} 40.8^{\prime \prime}$ & 3 & Ponkan \\
\hline 1.3.4.2 & $\mathrm{D}$ & Pomar7 & Inconfidentes & $046^{\circ} 17^{\prime} 33.8^{\prime \prime}$ & $22^{\circ} 17^{\prime} 42.4^{\prime \prime}$ & 3 & Ponkan \\
\hline 1.3.4.3 & $\mathrm{D}$ & Pomar7 & Inconfidentes & $046^{\circ} 17^{\prime} 31.9^{\prime \prime}$ & $22^{\circ} 17^{\prime} 42.5^{\prime \prime}$ & 3 & Ponkan \\
\hline
\end{tabular}


Também foram verificadas as características internas presente nos pomares que podem influenciar a presença e a infestação do inseto obtidas por meio de uma conversa informal com os produtores e visita nos pomares. Em que foram identificadas características referentes a: (i) presença de planta hospedeira, (ii) presença de açudes/ rios, (iii) presença de matas, (iv) uso de inseticidas e (v) presença de culturas próximas (Tabela 2).

\section{Monitoramento de Diaphorina citri nas plantas de murta}

Para a verificação de ninfas, ovos e adultos do inseto nas plantas de 'murta' das vias públicas do Município de Inconfidentes - MG (Figura 2) foram feitas inspeções visuais, selecionando 32 árvores de Murta presentes no

Tabela 2. Características internas dos pomares selecionados (Inconfidentes, Minas Gerais)

\begin{tabular}{|c|c|c|c|c|c|c|c|c|}
\hline \multirow{3}{*}{ Pomar } & \multicolumn{8}{|c|}{ Características } \\
\hline & \multicolumn{2}{|c|}{ Mata } & \multicolumn{2}{|c|}{ Rio/Açude } & \multirow{2}{*}{ P. Hospedeira } & \multirow{2}{*}{ Inseticida } & \multirow{2}{*}{$\begin{array}{l}\text { Culturas } \\
\text { próximas }\end{array}$} & \multirow{2}{*}{ Greening } \\
\hline & Sim & Não & Sim & Não & & & & \\
\hline 1 & Toda borda & & $\mathrm{X}$ & & Ausente & Sim & Ausente & Sim \\
\hline 2 & & $X$ & $\mathrm{X}$ & & Ausente & Não & $\begin{array}{l}\text { Café, } \\
\text { Banana }\end{array}$ & Não \\
\hline 3 & Nas laterais & & & $\mathrm{X}$ & Ausente & Não & $\begin{array}{c}\text { Café, } \\
\text { Maracujá }\end{array}$ & Sim \\
\hline 4 & Toda borda & & $X$ & & Ausente & Não & Uva & Não \\
\hline 5 & Nas laterais & & $X$ & & Ausente & Não & Ausente & Não \\
\hline 6 & & $X$ & $\mathrm{X}$ & & Presente & Não & $\begin{array}{l}\text { Uva, } \\
\text { Maracujá, } \\
\text { Café, } \\
\text { Banana }\end{array}$ & Sim \\
\hline 7 & Nas laterais & & $\mathrm{X}$ & & Ausente & Não & Uva & Não \\
\hline
\end{tabular}

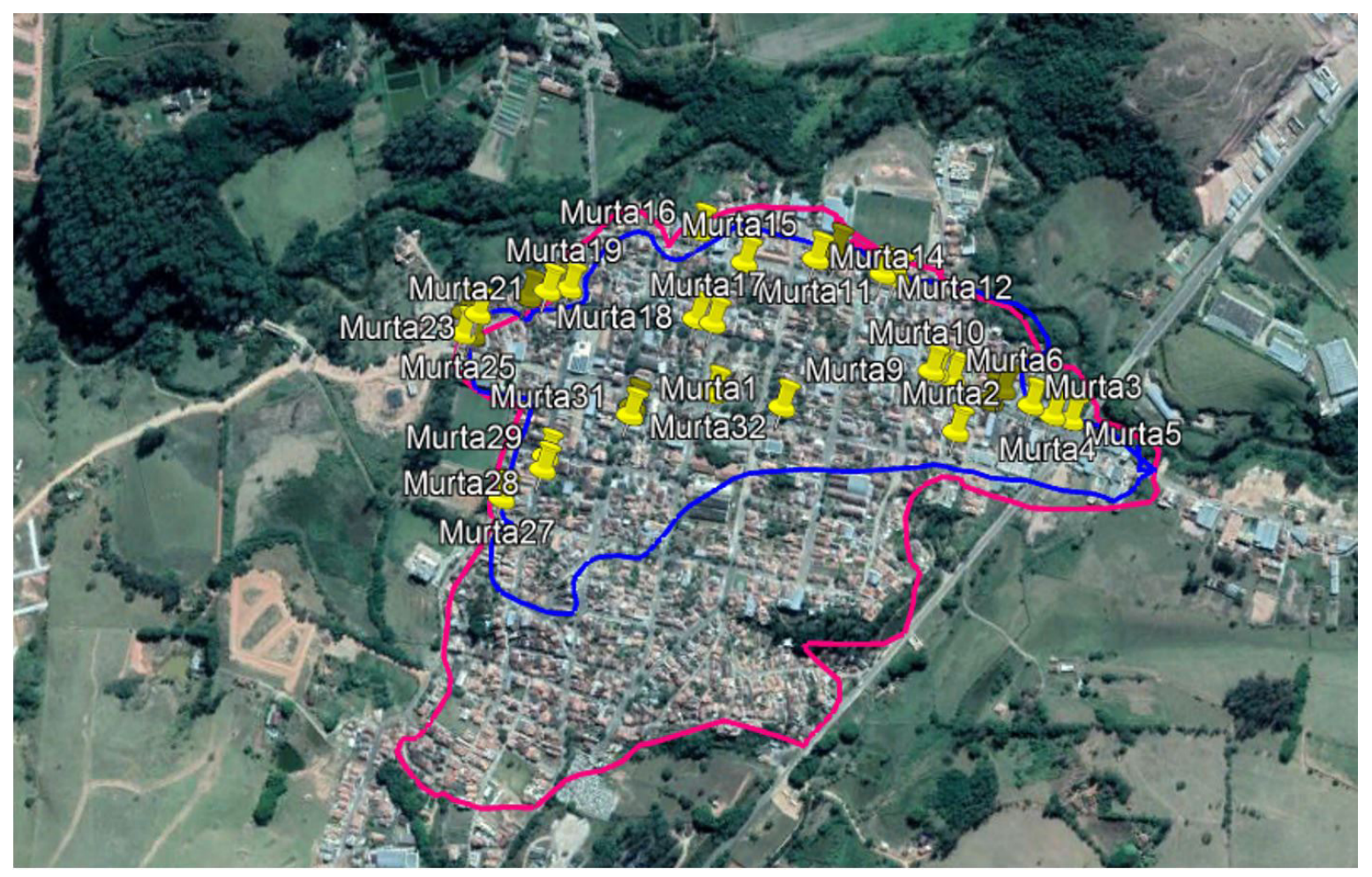

Figura 2. Localização das plantas de 'murta' nas vias públicas do município de Inconfidentes, MG. (i) Linha rosa delimitando o município; (ii) linha azul delimitando a área com presença de plantas de murta. 
paisagismo urbano do Município, com base nos locais de maior movimentação de pessoas e comércio na cidade, pois acredita-se que o inseto possa se translocar de um pomar ao outro por meio do transporte de frutas ou mudas cítricas decorrentes do estacionamento embaixo das árvores de murtas.

Foram coletadas 10 brotações de cada planta de murta, totalizando 320 brotações. Estas brotações foram levadas ao laboratório de Agroecologia, onde foram identificados e quantificados ovos e ninfas do inseto. Estas coletas foram repetidas a cada 15 dias, no período de Maio a Setembro de 2018.

A coleta do inseto adulto foi realizada com o uso de um sugador bucal improvisado utilizando um tubo de vidro transparente fechado com uma rolha, contendo dois orifícios, ao qual, foram encaixados dois canos de aquário transparentes de comprimento de $50 \mathrm{~cm}$ cada (Figura 3).

Um dos orifícios foi vedado com um tecido de textura fina, de forma que agisse como vedação a passagem do inseto no ato da sugação. Todas as 32 plantas de 'murta', foram georeferenciadas (Tabela 3).

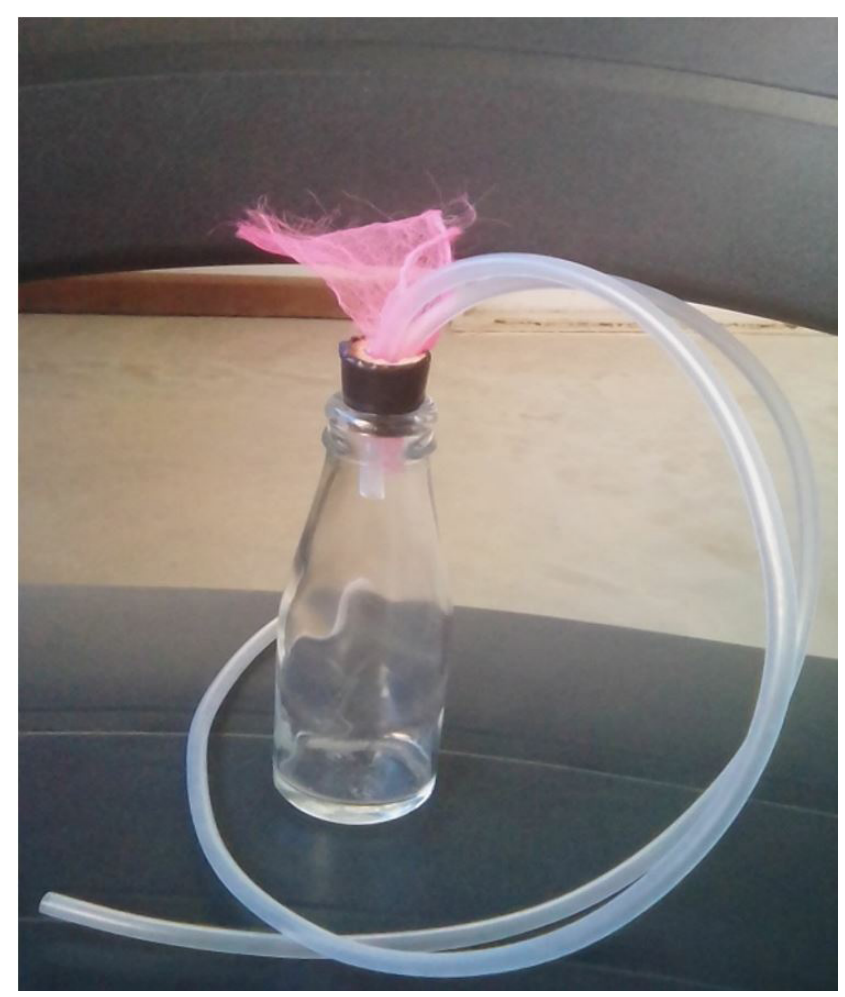

Figura 3. Sugador bucal improvisado utilizado no experimento, para coleta de Diaphorina citri (Inconfidentes, MG).

\section{ANÁLISE DOS DADOS ESTATÍSTICOS}

\section{Avaliação do nível de infestação de $D$. Citri}

Para verificar o nível de infestação de $D$. citri nas plantas de 'murta' presentes nas vias públicas do município de Inconfidentes-MG e nos pomares citrícolas, usou-se o programa Arc-gis Pro, em que as coordenadas geográficas das plantas de murta foram transformadas em números decimais por meio do uso de uma calculadora geográfica,

Tabela 3. Coordenadas das plantas de 'murta' do munício de Inconfidentes, Minas Gerais

\begin{tabular}{|c|c|c|}
\hline \multirow{2}{*}{ Plantas } & \multicolumn{2}{|c|}{ Coordenadas } \\
\hline & $\mathrm{S}$ & W \\
\hline Murta 1 & $22^{\circ} 19^{\prime} 4.67^{\prime \prime}$ & 4619'39.21" \\
\hline Murta 2 & $22^{\circ} 19^{\prime} 5.82^{\prime \prime}$ & $46^{\circ} 19^{\prime} 30.20^{\prime \prime}$ \\
\hline Murta 3 & $22^{\circ} 19^{\prime} 6.03 ”$ & $46^{\circ} 19^{\prime} 27.66^{\prime \prime}$ \\
\hline Murta 4 & $22^{\circ} 19^{\prime} 5.06^{\prime \prime}$ & $46^{\circ} 19^{\prime} 25.12^{\prime \prime}$ \\
\hline Murta 5 & $22^{\circ} 19^{\prime} 5.27^{\prime \prime}$ & 46'19'24.17' \\
\hline Murta 6 & $22^{\circ} 19^{\prime} 3.33^{\prime \prime}$ & $46^{\circ} 19^{\prime} 30.36^{\prime \prime}$ \\
\hline Murta 7 & $22^{\circ} 19^{\prime} 3.84^{\prime \prime}$ & 46¹9'27.69"' \\
\hline Murta 8 & $22^{\circ} 19^{\prime} 4.37^{\prime \prime}$ & $46^{\circ} 19^{\prime} 28.33^{\prime \prime}$ \\
\hline Murta 9 & $22^{\circ} 19^{\prime} 3.03^{\prime \prime}$ & $46^{\circ} 19^{\prime} 31.24^{\prime \prime}$ \\
\hline Murta 10 & $22^{\circ} 19^{\prime} 2.98^{\prime \prime}$ & $46^{\circ} 19^{\prime} 31.46^{\prime \prime}$ \\
\hline Murta 11 & $22^{\circ} 18^{\prime} 58.59^{\prime \prime}$ & $46^{\circ} 19^{\prime} 33.25^{\prime \prime}$ \\
\hline Murta 12 & $22^{\circ} 18^{\prime} 58.41^{\prime \prime}$ & $46^{\circ} 19^{\prime} 33.94$ " \\
\hline Murta 13 & $22^{\circ} 18^{\prime} 57.36^{\prime \prime}$ & 46¹9'36.40" \\
\hline Murta 14 & $22^{\circ} 18^{\prime} 57.73^{\prime \prime}$ & $46^{\circ} 19^{\prime} 37.43^{\prime \prime}$ \\
\hline Murta 15 & $22^{\circ} 18^{\prime} 57.90^{\prime \prime}$ & $46^{\circ} 19^{\prime} 41.13^{\prime \prime}$ \\
\hline Murta 16 & $22^{\circ} 18^{\prime} 56.38^{\prime \prime}$ & $46^{\circ} 19^{\prime} 43.48^{\prime \prime}$ \\
\hline Murta 17 & $22^{\circ} 19^{\prime} 0.74^{\prime \prime}$ & $46^{\circ} 19^{\prime} 42.76^{\prime \prime}$ \\
\hline Murta 18 & $22^{\circ} 19^{\prime} 0.55^{\prime \prime}$ & $46^{\circ} 19^{\prime} 43.64^{\prime \prime}$ \\
\hline Murta 19 & $22^{\circ} 18^{\prime} 59.10^{\prime \prime}$ & $46^{\circ} 19^{\prime} 50.23^{\prime \prime}$ \\
\hline Murta 20 & $22^{\circ} 18^{\prime} 59.02^{\prime \prime}$ & $46^{\circ} 19^{\prime} 50.60 "$ \\
\hline Murta 21 & $22^{\circ} 18^{\prime} 59.21^{\prime \prime}$ & $46^{\circ} 19^{\prime} 51.33^{\prime \prime}$ \\
\hline Murta 22 & $22^{\circ} 18^{\prime} 59.50^{\prime \prime}$ & $46^{\circ} 19^{\prime} 52.35^{\prime \prime}$ \\
\hline Murta 23 & $22^{\circ} 19^{\prime} 0.27^{\prime \prime}$ & $46^{\circ} 19^{\prime} 54.96^{\prime \prime}$ \\
\hline Murta 24 & $22^{\circ} 19^{\prime} 0.21^{\prime \prime}$ & $46^{\circ} 19^{\prime} 55.65^{\prime \prime}$ \\
\hline Murta 25 & $22^{\circ} 19^{\prime} 1.21^{\prime \prime}$ & 46'19'55.77' \\
\hline Murta 26 & $22^{\circ} 19^{\prime} 1.27^{\prime \prime}$ & $46^{\circ} 19^{\prime} 55.26^{\prime \prime}$ \\
\hline Murta 27 & $22^{\circ} 19^{\prime} 8.94 "$ & $46^{\circ} 19^{\prime} 53.68^{\prime \prime}$ \\
\hline Murta 28 & $22^{\circ} 19^{\prime} 7.57^{\prime \prime}$ & $46^{\circ} 19^{\prime} 51.56^{\prime \prime}$ \\
\hline Murta 29 & $22^{\circ} 19^{\prime} 6.95^{\prime \prime}$ & $46^{\circ} 19^{\prime} 51.42^{\prime \prime}$ \\
\hline Murta 30 & $22^{\circ} 19^{\prime} 4.64 ”$ & $46^{\circ} 19^{\prime} 46.90^{\prime \prime}$ \\
\hline Murta 31 & $22^{\circ} 19^{\prime} 5.04^{\prime \prime}$ & $46^{\circ} 19^{\prime} 47.05^{\prime \prime}$ \\
\hline Murta 32 & $22^{\circ} 19^{\prime} 4.02^{\prime \prime}$ & $46^{\circ} 19^{\prime} 42.77^{\prime \prime}$ \\
\hline
\end{tabular}


para que fosse possível fazer o georeferenciamento das coordenadas no software.

Posteriormente, foi calculada a média aritmética dos dados coletados para que fosse possível usar a ferramenta de Interpolação pela Ponderação do Inverso da Distância (IDW) do software Arcgis para se fazer o mapa do nível de infestação do inseto.

A Interpolação pela Ponderação do Inverso da Distância é determinada pela equação matemática:

$$
\frac{z=\sum_{i=1}^{n} \frac{1}{d_{i}} z_{i}}{\sum_{i=1}^{n} \frac{1}{d_{i}}}
$$

Em que $\mathrm{z}$ são os valores estimados, $\mathrm{n}$, o número de amostras, zi os valores conhecidos e di a distância dos valores conhecidos e estimados (zi e z).

\section{RESULTADOS E DISCUSSÃO}

Foi constatada a presença do inseto vetor do greening D. citri nas plantas de 'murta' presentes nas vias públicas do município de Inconfidentes-MG, sendo quantificados ao todo 27 adultos, 238 ninfas e 221 ovos, em que o mês de maior amostragem do inseto foi Setembro (Figura 4).

A maior concentração de ninfas de $D$. citri estava presente nas plantas de 'murtas' próximas a pontos comerciais, em que, as cores mais escuras presentes no mapa, representam os maiores índices de concentração das ninfas, representado pelas murtas 10, 5, 21 e 28 com médias variando de 1 a 5 ninfas por planta (Figura 5).

O maior índice de infestação de ovos foi constado nas murtas 5 e 6 com médias de 7 ovos por planta (Figura 6).

Embora o nível de infestação de ninfas e ovos seja de médio a alto, a infestação de insetos adultos se manteve

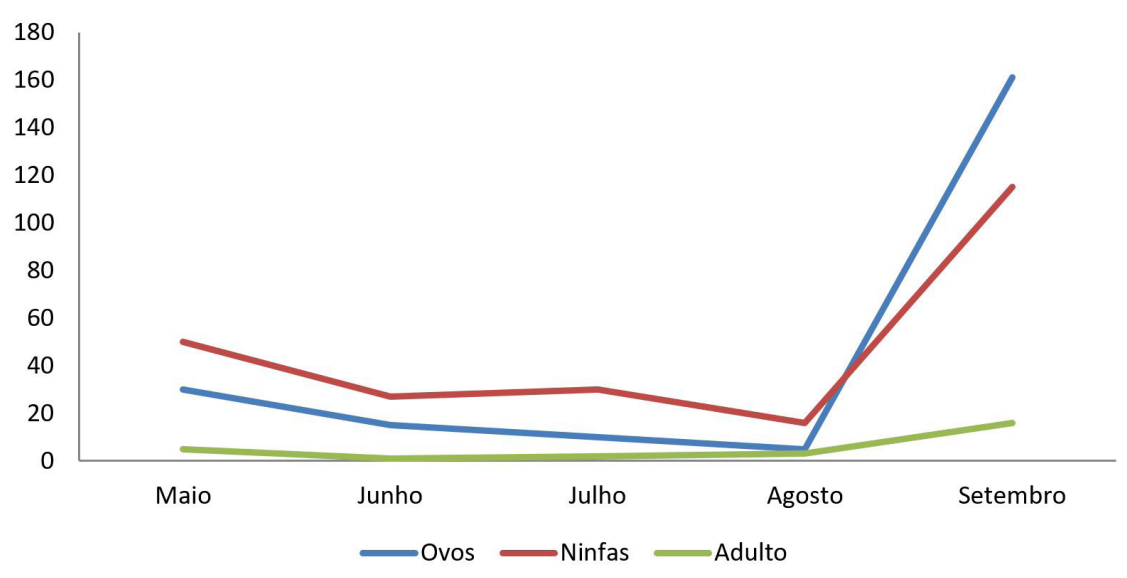

Figura 4. Representação da quantificação dos estádios de desenvolvimento de $D$. citri em relação ao mês de coleta (Inconfidentes, Minas Gerais).
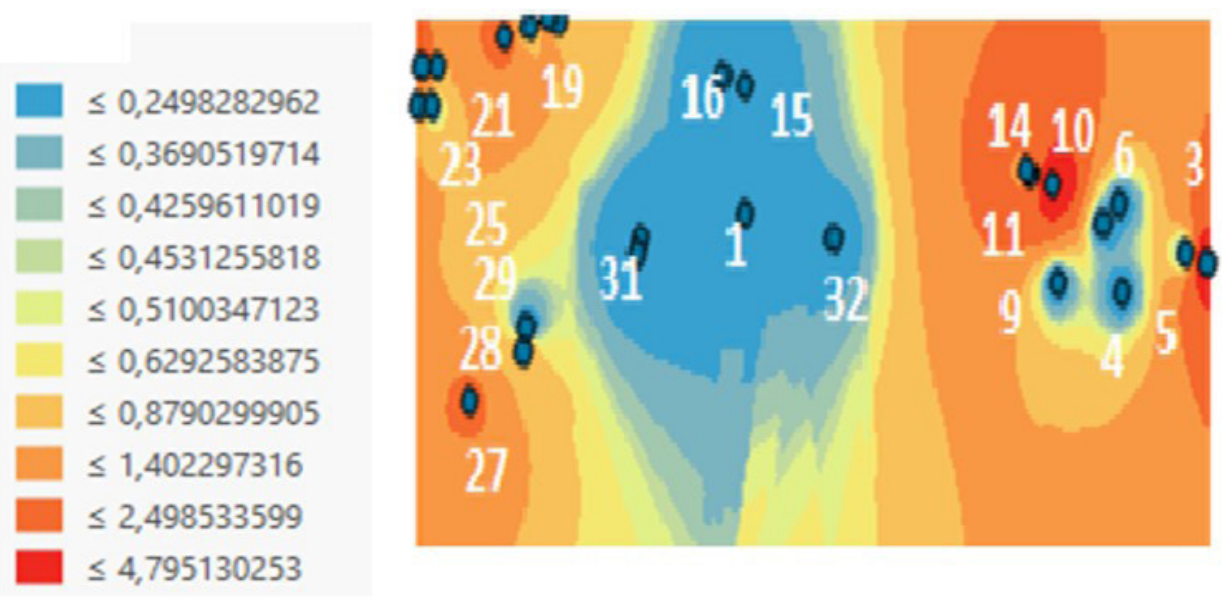

Figura 5. Mapa representativo do nível de infestação, expresso em número de ninfas de Diaphorina citri, presente nas brotações analisadas de exemplares de Murraya paniculata (Inconfidentes, Minas Gerais). 

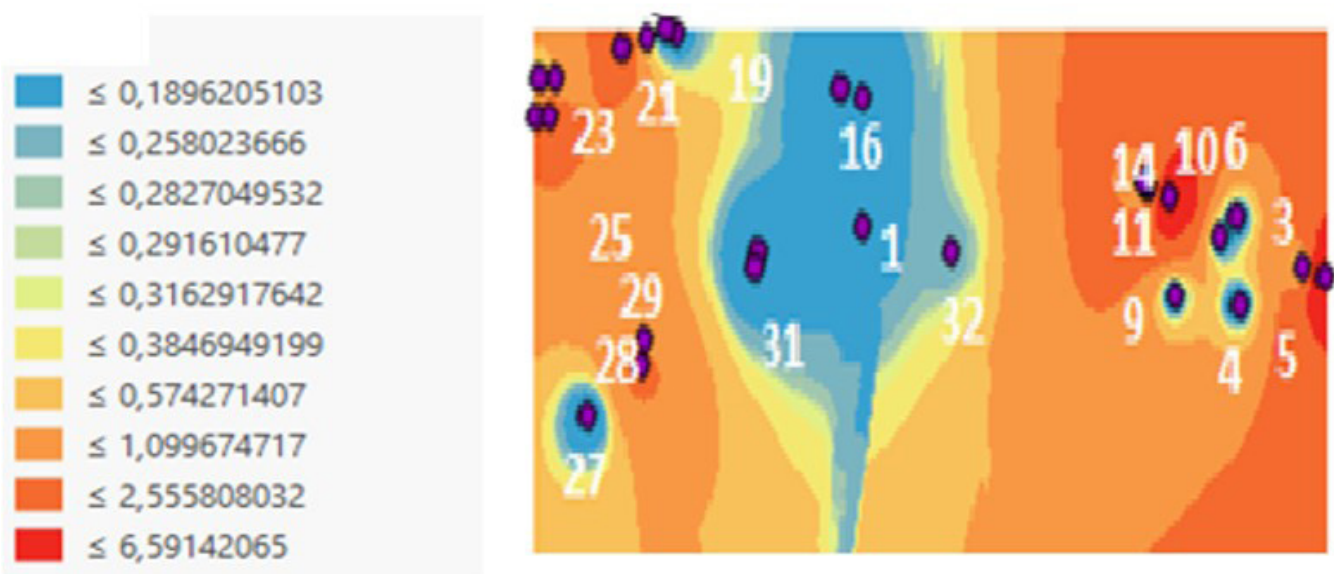

Figura 6. Mapa representativo do nível de infestação de ovos de Diaphorina citri presentes nas brotações analisadas de exemplares de Murraya paniculata (Inconfidentes, Minas Gerais ).
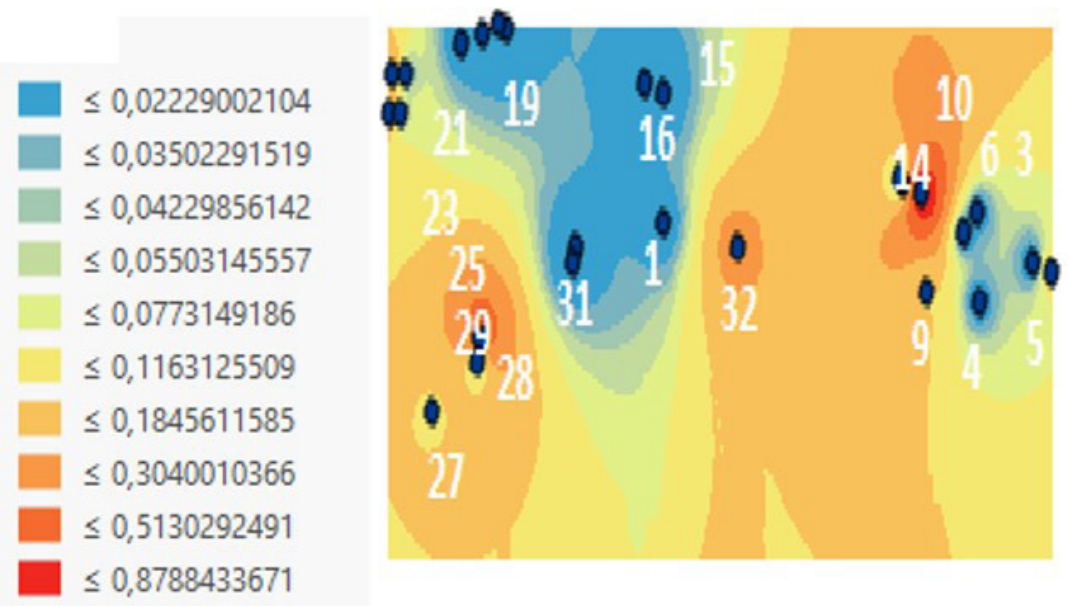

Figura 7. Mapa representativo do nível de infestação de adultos de Diaphorina citri presentes nas brotações analisadas de exemplares de Murraya paniculata (Inconfidentes, Minas Gerais ).

baixa quando comparado com o estudo de Yamamoto et al. (2001), em que foi observado no mês de setembro de 1994 na região de Barretos-SP, aproximadamente 60 insetos adultos por minuto de sucção. Este resultado se deve provavelmente em decorrência do período de ausência de reprodução do inseto adulto e da presença de poucas brotações. Contudo, foi registrado a presença de aproximadamente um inseto por planta, observado na murta 4 (Figura 7).

A presença das plantas de 'murta' nas vias públicas do município de Inconfidentes, pode estar relacionado com a falta de fiscalização frente aos viveiros que comercializam mudas da planta. Em que, apesar de ser proibido o seu plantio e comércio no Estado de Minas Gerais, determinado pela Portaria $\mathrm{N}^{\circ} 1649$ de 18 de agosto de 2016 (Minas Gerais, 2016), ainda sim muitos viveiros detém do exemplar. E embora, seja indicado a aplicação de inseticidas nas plantas cítricas precedendo a sua retirada do viveiro, o mesmo não acontece com as plantas de murta. Assim sem a eliminação do inseto, este provavelmente, veio junto com o transporte para a cidade.

Contudo, apesar de ser constada a presença do vetor D. citri no município de Inconfidentes- $M G$, não foi detectada a presença do inseto nos pomares citrícolas, perante a analise das armadilhas e brotações, mesmo a doença ter sido identificada nos pomares na faixa etária de 13 anos.

Já os pomares na faixa etária de 23 a 25 anos não apresentaram diagnóstico positivo à doença podendo estar relacionado ao fato que as plantas com idades mais avançadas tendem a demorar mais para manifestar os sintomas quando contaminadas (Fundo de Defesa da Citricultura, 2017). 
E segundo a Fundecitrus (Fundo de Defesa da Citricultura, 2017), a maior prevalência de plantas sintomáticas se dá em plantas na faixa etária acima de 10 anos, tornando-se comum a detecção das bactérias causadoras do greening nas plantas nesta faixa etária.

Todavia, após a primeira detecção da doença no pomar, novas plantas podem vir a manifestar os sintomas, isso porque a amostragem das folhas para detecção das bactérias não é $100 \%$ eficaz, pois a concentração das bactérias na planta se apresenta de forma irregular, em que muitas plantas infectadas não são detectáveis pelo teste de PCR (Bassanezi et al., 2010), explicando assim, novas e futuras erradicações nos pomares a medida que novas fiscalizações são realizadas, pois a retirada das plantas infectadas no pomar tem sido a forma mais indicada de se evitar que a doença se dissemine.

E apesar dos pomares de 1 e 3 anos, não apresentarem resultados positivos a doença segundo os exames laboratoriais cedidos pelo IMA não podemos descartar o risco, uma vez, que a patologia fica incubada e a medida que se aumenta a concentração da bactéria no floema, os sintomas se tornam mais evidentes pelo PCR.

Assim, a não detecção do inseto pelas armadilhas adesivas, mesmo que estes tenham sido registrados nas plantas de murta presentes nas vias públicas do município, pode estar associado ao pouco movimento efetivo do inseto (Tomaseto, 2012).

\section{CONCLUSÕES}

Os resultados obtidos com o trabalho é um alerta para uma possível infestação do Diaphorina citri em pomares citrícolas da região, que mesmo não tendo sido detectado nos pomares citrícolas pelo estudo, não está descartada a possibilidade.

A presença do vetor do greening nas plantas de murta do município e sua não detecção nos pomares citrícolas, indica a necessidade de monitoramento periódico dos pomares, além de maior exigência dos órgãos fiscalizadores da doença. Evitando o plantio e realizando a substituição das plantas de murta, quando possível no município.

\section{REFERÊNCIAS}

Atihe Junior, J. A., Pino, A. F., Medonça, E. T. \& Francisco, V. L. F. S. (2006) Incidência de Huanglongbing (HLB) (greening) em citros na região de Araraquara. Laranja, 27(2), 251-262.
Bassanezi, R. B., Lopes, S. A., Belasque, J., \& Sposito, M. B. (2010). Epidemologia do Huanglongbing e suas implicações para o manejo da doença. Citrus Research \& Technology, 31(1), 11-23.

Burckhardt, D., \& Ouvrard, D. (2012). A revised classification of the jumping plant-lice (Hemiptera: Psylloidea). Zootaxa, 3509(1), 1-34.

Coletta, F. H. D., Targon, M. L. P. N., Takita, M. A., \& Negri, J. D. (2004). First reporto $f$ the causal agente of huanglongbing (Candidatus Liberibacter asiaticus) in Brazil. Plant Disease, 88, 1382.

Empresa Brasileira de Pesquisa Agropecuária-EMBRAPA. (2016). Produção Brasileira de Tangerina em 2016. Recuperado em 19 de abril de 2018, de http://www. cnpmf.embrapa.br/Base_de_Dados/index_pdf/dados/ brasil/tangerina/b32_tangerina.pdf

Erpen, L., Muniz, F. R., Moraes, T. S., \& Tavano, E. C. R. (2018). Análise do cultivo da laranja no Estado de São Paulo de 2001 a 2015. Revista IPecege, 4(1), 33-43.

Fernandes, N. G. (2004). Combate ao greening em citros necessita de legislação específica. Visão Agrícola, 2(1), 40-43.

Fundo de Defesa da Citricultura - FUNDECITRUS. (2017). Levantamento de Plantas sintomáticas do greening de acordo com a idade da planta. Recuperado em 7 de agosto de 2018, de http://www.fundecitrus.com.br/ levantamentos/greening

Minas Gerais. Ministério da Agricultura, Pecuária e Abastecimento. (2011). Ato Portaria $n^{\circ} 344$, de 29 de Agosto de 2011. Aprova o Zoneamento Agrícola de Risco Climático para a cultura de citros no Estado de Minas Gerais. Brasília, DF: Diário Oficial da República Federativa do Brasil, seção 1. Recuperado em 11 de janeiro de 2018, de http://www.agricultura.gov.br/assuntos/riscos-seguro/ risco-agropecuario/portarias/safra-vigente/minas-gerais/ word/PORTN344ZONAGR11CITROSMG.rtf

Minas Gerais. Governo do Estado. Instituto Mineiro de Agropecuária. (2016). Portaria $n^{\circ} 1649$, de 18 de agosto de 2016. Revoga as Portarias $n^{\circ} 1644 / 2016$ e n $n^{\circ}$ 1227/2012, estabelece procedimentos complementares e delimita as áreas para adoção de medidas de prevenção e erradicação do Greening em Minas Gerais. Belo Horizonte, MG: Diário Oficial do Estado de Minas Gerais.

Paiva, P. E. B. (2009). Distribuição espacial e temporal, inimigos naturais e tabela de vida ecológica de Diaphorina 
citri Kuwayama (Hemiptera: Psyllidae) em citros em São Paulo (Tese de doutorado). Escola Superior de Agricultura "Luiz de Queiroz, Universidade de São Paulo, Piracicaba.

Parra, J. R. P., Lopes, J. R. S., Torres, M. L. G., Nava, D. E., \& Paiva, P. E. B. P. (2010). Bioecologia do vetor Diaphorina citri e transmissão de bactérias associadas ao huanglongbing. Citrus Research \& Technology, 31(1), $37-51$.

Tomaseto, A. F. (2012). Capacidade de dispersão de Diaphorina citri Kuwayama (Hemiptera: Psyllidae). (Dissertação de mestrado). Escola Superior de Agricultura "Luíz de Queiroz", Universidade de São Paulo, Piracicaba.

Tomaseto, A. F. (2016). Fatores bióticos e abióticos influenciando a mobilidade e aspectos biológicos de Diaphorina citri Kuwayama (Hemiptera: Liviidae) (Tese de doutorado). Escola Superior de Agricultura "Luiz de Queiroz”, Universidade de São Paulo, Piracicaba.
Yamamoto, P. T., Paiva, P. E. B., \& Gravena, S. (2001). Flutuação populacional de Diaphorina citri Kuwayama (Hemiptera: Psyllidae) em pomares de citros na Região Norte do Estado de São Paulo. Neotropical Entomology, 30(1), 165-170.

Yamamoto, P. T; Alves, G. R \& Beloti, V. H.(2015) Manejo e controle do huanglongbing (HLB) dos cítricos. Investigación Agraria,16(2).

Recebido: Fevereiro 19, 2019

Aceito: Outubro 31, 2019

Como citar: Silva, B. M., Pereira, J. M., \& Rocha, L. C. D. (2019). Nível de infestação de Diaphorina citri no Município de Inconfidentes, Minas Gerais. Citrus Research \& Technology, 40, e1052. https://doi.org/10.4322/crt.18519 